\title{
NEARLY SIMULTANEOUS OPTICAL AND VLBI POLARIZATION OBSERVATIONS OF BL LAC OBJECTS
}

\author{
D.C. GABUZDA AND P.Y. KOCHANEV \\ Lebedev Physical Institute, Moscow, RUSSIA \\ M.L.SITKO \\ University of Cincinnati, Cincinnati, Ohio, USA \\ AND \\ P.S. SMITH \\ University of Arizona, Tucson, Arizona, USA
}

\section{Introduction}

The continua of BL Lacertae objects and other "blazars" are dominated by nonthermal emission that is variable and highly polarized at UV-radio wavelengths (Angel and Stockman 1980; Kollgaard 1994; Allen et al. 1993). It is believed that this non-thermal emission is associated with the relativistic jets that are known to exist in these sources, but details of the jet structure and physics are still very uncertain. It is usually expected that the polarization behavior at optical and radio wavelengths should show little or no correlation, even if genuinely simultaneous measurements are compared. It is typically thought that the emission in these two wavebands originates in vastly different parts of the source, where the magnetic field geometries are likely to be quite different. In some inhomogeneous synchrotron source models for blazars, however, depending on the model parameters considered, the radio and UV-optical-IR (UVOIR) emission may be co-spatial (Ghisellini, Maraschi \& Treves (1985)). It is thus of interest to search for correlations between the emission of blazars in the UVOIR and radio, to test such models. Our approach to doing this has been to compare simultaneous measurements of the optical and VLBI polarization characteristics of compact AGN. The polarization of the radiation is effectively used as a probe of the magnetic field structures in the regions where the emission at the two wavelengths arises. 


\section{Results}

We will use $\chi_{o p t}, \chi_{c o r e}$, and $\chi_{j e t}$ to refer to the optical, VLBI core, and VLBI jet polarization position angles, respectively. Simultaneous measurements of the optical and VLBI polarization for three BL Lacertae objects have recently been analyzed. Comparison of optical and VLBI polarization for five other blazars was presented earlier by Gabuzda \& Sitko (1994). In every source, there has been a clear association between $\chi_{\text {opt }}$ and $\chi$ in one of the VLBI components. In six of the eight sources, $\chi_{\text {opt }}$ is correlated with $\chi_{\text {core }}$, in one case (OJ 287) clearly in association with the birth of a new VLBI component; in the remaining two sources, $\chi_{o p t}$ was correlated with $\chi_{j e t}$ in a recently emerged new VLBI component very close to the core. Thus, these comparisons point toward a correlation between the optical polarization and the polarization in the VLBI cores and in young, recently emerged VLBI jet components in BL Lacertae objects. This idea is consistent with the suggestion by Gabuzda et al. (1994; see also Gabuzda, Pushkarev, \& Cawthorne, these proceedings) that the $6 \mathrm{~cm}$ VLBI core polarizations of BL Lacertae objects tend to be dominated by the polarization of new jet components that are not yet resolved from the core.

A more thorough presentation and discussion of these data will be given in a paper by Gabuzda, Sitko, and Smith, to be submitted to the Astronomical Journal in November, 1995.

\section{Ongoing Work}

Simultaneous optical and VLBI polarization data for 10 BL Lacertae objects were obtained in May-August, 1995. These observations will bring the total number of sources for which such data are available to 15. Any optical-VLBI polarization correlations should be much more clearly seen in this larger sample. Work with a larger sample will also enable us to begin comparison of the optical and VLBI degrees of polarization, which we have not yet attempted with our smaller number of sources. We will continue trying to obtain such data, particularly for VLBI experiments at shorter $\mathrm{cm}$ wavelengths. Another interesting question that remains to be investigated is whether there is evidence for optical-VLBI polarization correlations for quasars as well as for BL Lacertae objects.

\section{References}

Allen et al. (1993) Astrophysical Journal, 403, 610.

Angel \& Stockman (1980) Annual Reviews of Astronomy and Astrophysics. 8, 321.

Gabuzda \& Sitko (1994), Astronomical Journal, 107, 884.

Ghisellini, Maraschi \& Treves (1985) Astronomy and Astrophysics. 146, 204.

Kollgaard (1994) Vistas in Astronomy. 38, 29. 